\title{
FuzzySTAR: Fuzzy set theory of axiomatic design review
}

\author{
GEORGE Q. HUANG ${ }^{1}$ AND ZUHUA JIANG ${ }^{2}$ \\ ${ }^{1}$ University of Hong Kong, Hong Kong, People's Republic of China \\ ${ }^{2}$ School of Mechanical Engineering, Shanghai Jiao Tong University, Shanghai, People's Republic of China \\ (Received September 1, 2001; Accepted May 26, 2002)
}

\begin{abstract}
Product development involves multiple phases. Design review (DR) is an essential activity formally conducted to ensure a smooth transition from one phase to another. Such a formal DR is usually a multicriteria decision problem, involving multiple disciplines. This paper proposes a systematic framework for DR using fuzzy set theory. This fuzzy approach to DR is considered particularly relevant for several reasons. First, information available at early design phases is often incomplete and imprecise. Second, the relationships between the product design parameters and the review criteria cannot usually be exactly expressed by mathematical functions due to the enormous complexity. Third, DR is frequently carried out using subjective expert judgments with some degree of uncertainty. The DR is defined as the reverse mapping between the design parameter domain and design requirement (review criterion) domain, as compared with Suh's theory of axiomatic design. Fuzzy sets are extensively introduced in the definitions of the domains and the mapping process to deal with imprecision, uncertainty, and incompleteness. A simple case study is used to demonstrate the resulting fuzzy set theory of axiomatic DR.
\end{abstract}

Keywords: Axiomatic Design; Design Review; Fuzzy Sets; Product Development

\section{INTRODUCTION}

Axiomatic design was originally proposed by Suh in the 1980 s, and it was later formulated as a generic theory of axiomatic design (TAD) as demonstrated systematically in Suh (1990). Since then, the method has gained wide recognition in both the research and industrial communities. Typical applications include mechanical products (Park et al., 1996; Cha \& Cho, 1999), software products (Kim et al., 1991; Chen, 1998), manufacturing systems (Suh et al., 1978), and design for environment (Wallace \& Suh, 1993; Chen, 2001). Recently, the method has been implemented as a commercial software package (Harutunian et al., 1996; http://www.axiomaticdesign.com).

In TAD, Suh (1990) defines design as the mapping process between the functional requirements (FRs) in the functional domain and the design parameters (DPs) in the physical domain. Conceptually, the design process can be interpreted as a process involving choosing the right set of

Reprint requests to: Department of Industrial and Manufacturing Systems Engineering, University of Hong Kong, Pokfulam Road, Hong Kong, PRC. E-mail: gqhuang@hkucc.hku.hk
DPs to satisfy the given FRs. Mathematically, the mapping is expressed as

$$
\text { design: }\{\mathbf{F R}\} \rightarrow\{\mathbf{D P}\}
$$

or, more specifically,

$$
\{\mathbf{F R}\}=[\mathbf{A}] \times\{\mathbf{D P}\},
$$

where $\{\mathbf{F R}\}$ is the FR vector, $\{\mathbf{D P}\}$ is the DP vector, and $[\mathbf{A}]$ is the design matrix.

The above is only a very brief summary of TAD. Detailed discussions on TAD should be sought from the Suh's original work $(1990,1999)$. More introductory discussions on the method can be found at http://www.axiomaticdesign. com and http://axiom.mit.edu). Hintersteiner and Friedman (1999) and Tate (1999) demonstrate and examine various key issues regarding how TAD as an abstract mathematical model can be put into practical applications.

Axiomatic design guides designers in using all their existing design tools and software to arrive at a successful new design or to diagnose and correct an existing design. The latter application in diagnosis and correction has motivated the authors to explore the possibility of extending 
the TAD into a framework for design review (DR, Huang, 2002a). The resulting framework is called STAR (systematic theory for axiomatic DR), which has also been implemented as a prototype web application for setting up a DR portal (Huang, 2002b).

In essence, STAR is defined as the reverse mapping from the DP domain to the FR domain, that is,

$$
\text { review: }\{\mathbf{D P}\} \rightarrow\{\mathbf{F R}\}
$$

Here the FR domain should be generalized as the DR criteria, which is the DR criterion domain ( $\mathrm{RC}$ domain). The $\mathrm{RC}$ domain can be established from the functional (FRs) and other types of design requirements (DRs), that is, $\{\mathbf{R C}\} \subseteq\{\mathbf{D R}\}$.

The establishment of STAR contributes to the scarce literature on DR. The ad hoc DR practice (Ichida, 1989) can now be guided in a systematic way. Thus, such systematic DR practice is more likely to meet the requirements imposed by the ISO 9000 quality standard under which DR is mandatory (Schoonmaker, 1996). An extra benefit of a systematic DR framework is that computerized decision support systems with the latest internet and web technologies can be developed for professional applications in industry. This is a significant addition to the product data management technology, where DR has traditionally not been emphasized as much as engineering change management.

However, DR is never a clear-cut exercise. Vague and uncertain descriptions of DPs and DR criteria are often involved (Li \& Azarm, 2000). This has made the mathemat- ical calculation (quantitative evaluation) very difficult. Qualitative analysis is widely needed with linguistic and vague descriptions of the complex DR system (Cheng, 1999). Furthermore, the mapping results from STAR do not address the ultimate question that DR must answer: is the design good or bad and which parts are good, which parts are bad, and to what extent?

Whereas Helander and Lin (2000) incorporated fuzzy set theory into Suh's TAD, this paper aims to resolve the questions raised above by introducing fuzzy set theory into STAR to form FuzzySTAR (fuzzy set theory for axiomatic DR). Section 2 details the extension process from STAR to FuzzySTAR and associated research issues. Section 3 presents a case study to illustrate how the proposed FuzzySTAR can be applied in an industrial environment. Observations and implications are discussed in Section 4 to conclude the paper.

\section{FuzzySTAR}

FuzzySTAR is an extension of a previous version of the STAR by incorporating fuzzy set theory. STAR is derived from Suh's TAD. Naturally, the definition and description of FuzzySTAR share some common features and conventions with TAD and STAR. Most noticeably, the constructs of domains, mappings, and axioms are all used in FuzzySTAR. Figure 1 shows the overview of FuzzySTAR. Its major steps, including the key concepts and associated mathematics, will be discussed in detail in the rest of this section.

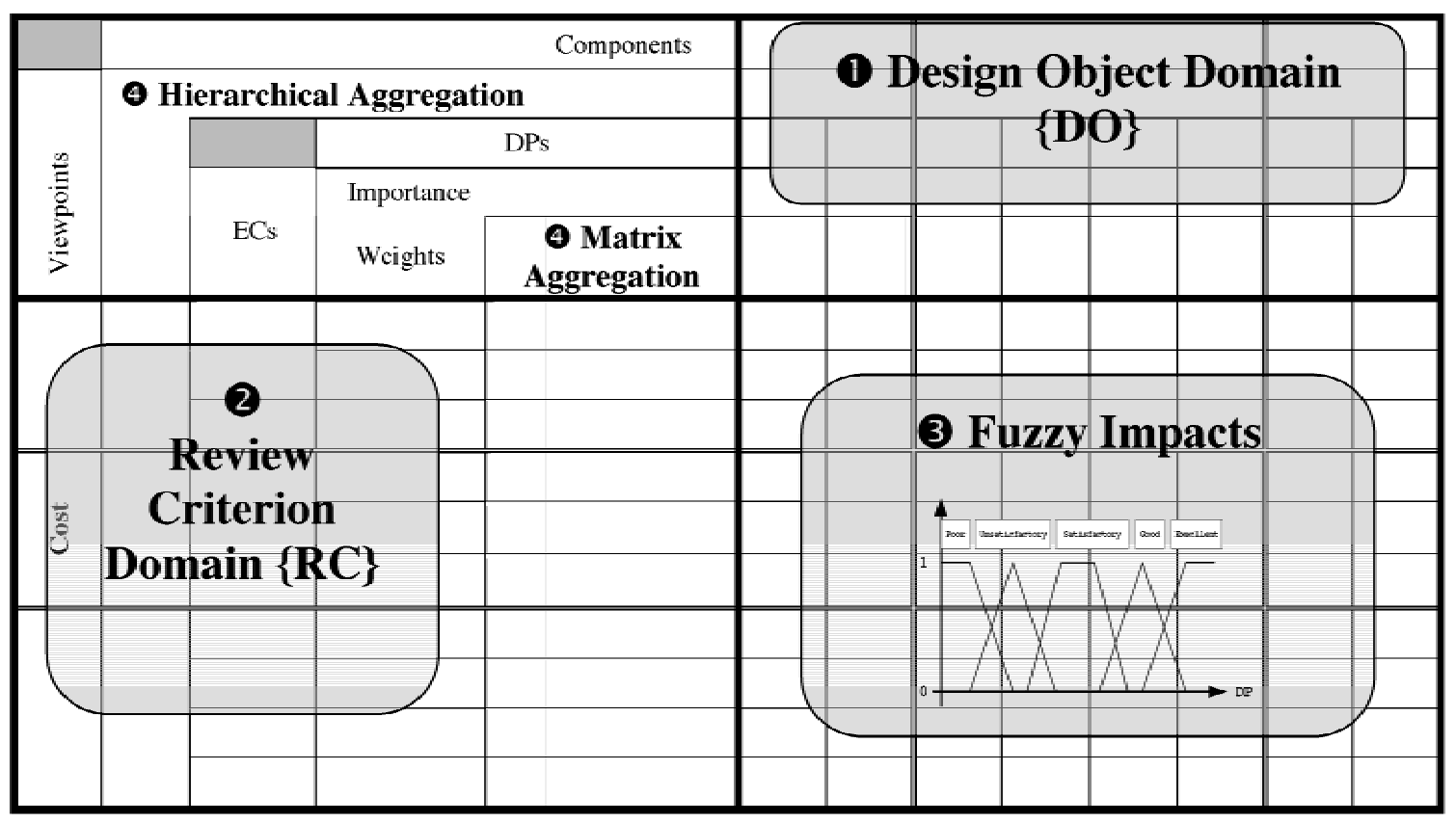

Fig. 1. An overview and the steps of FuzzySTAR. 


\subsection{Step 1: Define the design objects $\{$ DO $\}$ domain}

As in TAD and STAR, the product design domain must be defined first. Let $\{\mathbf{D O}\}$ be defined as a collection (domain or vector) of DOs that make up the product under review. Although the mathematical definition here seems to imply that the $\{\mathbf{D O}\}$ domain is a flat structure, the hierarchical tree representation is commonly used in practice. The levels from the top to the bottom in the DO hierarchy reflect the evolution of the design process from early stages to more detailed stages, on the one hand, and the shifting foci or concerns of the DR process, on the other.

A DO is defined as a triplet: DP, target value (TV), and design weight (DW). The first element in the DO triplet is a DP that describes and defines a product DO collectively with other DPs. The collection of all the DPs in the $\{\mathbf{D O}\}$ domain forms another DP domain $\{\mathbf{D P}\}$.

The second element of the DO triplet, TV, is the value (or range of values) of the corresponding DP. The collection of all the TVs in the $\{\mathbf{D O}\}$ domain is the new value domain $\{\mathbf{T V}\}$. The TVs of DPs can be defined as either crisp numbers or a region (range) of crisp numbers. At this stage of the work reported in this paper, only crisp values are considered and region values are avoided for simplicity.

The third element in the DO triplet, DW, is the weight or rating based on a certain aspect (e.g., the cost of changing the TV of the corresponding DP). The aspect of "changing a DP's value" is mentioned here in regard to modular design, in which some DPs cannot be changed if the DO is a module. In addition, the subsequent action following the DR is usually design revision or change. The collection of all the DWs forms the set of weights on DP when taking TV as its value.

The DWs are normally expressed as subjective crisp numbers (see, e.g., Khoo \& Ho, 1996; Moskowitz \& Kim, 1997). Very often, these weights are typically uncertain and imprecise. Therefore, they have also been expressed as fuzzy numbers (Masud \& Dean, 1993; Wang, 1999). Typical linguistic terms for describing grades of the cost of changing the value of a DP are difficult, possible, and easy.

DWs are usually established based on past technical experiences or expert judgments. It is outside the scope of this work to discuss how this can be accomplished. Several techniques are available to obtain these values, whether crisp or fuzzy. One of the most widely used methods is the analytical hierarchy process (AHP; e.g., Park \& Kim, 1998). Weights from AHP can be converted into fuzzy numbers based on the concept of the "fuzzy line segment" proposed by Carnahan et al. (1994).

\subsection{Step 2: Define the $\{\mathbf{R C}\}$ domain}

Let $\{\mathbf{R C}\}$ define a collection (domain or vector) of RCs used for reviewing the $\{\mathbf{D O}\}$. Just like the $\{\mathbf{D O}\}$ domain, the $\{\mathbf{R C}\}$ domain is commonly represented hierarchically, as a tree in practice. The higher levels are called "view- points" of DR. The RC at the bottom level are those directly used for evaluating the DOs. That is, the relationships between the DPs and the RC at the bottom level can be directly established and analyzed. The results are propagated or aggregated upward in the hierarchy as discussed in a later section.

A RC is also expressed as a triplet: evaluation criterion (EC), design capability (DC), and functional weight (FW). The first element in the $\{\mathbf{R C}\}$ domain is an EC. This is a description of an objective that DOs intend to achieve. The collection of all the RCs in the $\{\mathbf{R C}\}$ domain forms another EC domain $\{\mathbf{E C}\}$.

The second element of the RC triplet, DC, is the value (or range of values) of the corresponding EC. This value or range of values describes the capability of the DOs to meet the intended DRs. The collection of all the ECs in the $\{\mathbf{R C}\}$ domain is the new DC domain $\{\mathbf{D C}\}$.

The third element in the RC triplet, $\mathrm{FW}$, is the weight or rating that the DOs collectively achieve against this $\mathrm{RC}$ (or EC). The collection of all the FWs forms the set of weights on RC. FWs can be either crisp values or fuzzy numbers, depending on the type of number used in evaluating the EC-DP relationships. If the relationships are described in fuzzy numbers, FWs are naturally described in fuzzy numbers. Typical linguistic terms for describing grades of an EC are excellent, good, satisfactory, barely satisfactory, and unsatisfactory or simply good, fair, and unsatisfactory.

Finally, there are two ways of obtaining the FW of a RC. One method is to derive the FW from fuzzy relationships between DPs and the EC through certain aggregation algorithms. This method is called the direct fuzzy mapping method because the FW is obtained directly from the membership functions established between the EC across the TVs of the DPs.

The other method is to derive the FW from a membership function established for the EC based on its DC. This method is called indirect or tandem fuzzy mapping method. In the tandem approach to DR, it is necessary to define the relationships between the DPs and RCs mathematically so that the absolute values of RCs can be estimated as output variables with respect to DPs as input variables. The complexity of the DR problem usually makes such mathematical modeling practically impossible. Fortunately, the direct mapping method does not have this problem.

Both methods are necessary in practical applications. For example, the case study on the bus inner configuration design to be discussed in a later section uses the first method. The case study on fuel pump design uses the second method (Huang \& Jiang, 2002b). The methods can also be applied in combination.

\subsection{Step 3: Evaluate the impacts of DPs on ECs}

This step is the most fundamental step in FuzzySTAR. It creates the review workspace and forms the basis for car- 
rying out fuzzy analysis. This step includes the following activities:

1. Create DR space (matrix): the STAR mapping creates the 2-dimensional review space (or matrix), which is the so-called universe of discourse used in fuzzy evaluation.

2. Determine for each EC if a particular DP is related to this $E C$ : If yes, a checkmark is placed in the corresponding cell. Otherwise, the cell is left blank, indicating that this DP does not affect the EC. This EC is said to "not care" about the DP. In the case of "an uncaring" EC-DP relationship, no further consideration is necessary.

3. Evaluate the $\{\mathbf{D C}\}$ for each $R C$ across the $\{\mathbf{D O}\}$ domain: This activity is only necessary in the tandem mapping method and is not included in the direct mapping method. In the tandem mapping method, the DC must be fuzzificated just like the fuzzification of the impacts in the direct mapping method in point 4 .

4. Fuzzificate the impact of the DP on the EC. As advocated by several researchers (Cheng, 1999; Li \& Azarm, 2000; Wang, 2001), this paper also advocates the approach of capturing the DP-RC relationships through fuzzy sets. Typical linguistic terms for describing the grades of the relationship between a DP and an EC are strong, medium, and weak or excellent, good, satisfactory, unsatisfactory, and poor.

The activity of fuzzification is worth further discussions in two different situations. In the first situation of tandem fuzzy mapping, the RCs obtained are crisp numbers. For example, $\{\mathbf{R C}\}=\{($ cost $, \$ 150, ?)$, (weight, $20 \mathrm{~kg}, ?)\}$. Now the remaining question is if this cost of $\$ 150$ and the weight of $20 \mathrm{~kg}$ reflect a good design or bad design. To answer this question, it is then necessary to fuzzificate these crisp numbers into fuzzy measures FWs. That is, $\{\mathbf{R C}\}=\{$ cost, $\$ 150$, $(0.3,0.4,0.1))$, (weight, $20 \mathrm{~kg},(0.1,0.2,0.5))\}$. These fuzzy numbers will be defuzzificated later to establish the grades excellent, fair, or poor.

In the case of direct fuzzy mapping, the activity of fuzzification is conducted to each cell in the review matrix. For each cell of the DP-RC matrix, that is, each pair (EC, DP) where $\mathrm{EC} \in\{\mathbf{E C}\}$ and $\mathrm{DP} \in\{\mathbf{D P}\}$, a fuzzy set is established to represent the impact of DP on EC by searching the fuzzy knowledge base that is a collection of membership functions. If a corresponding membership function is found, then the fuzzy number is evaluated from the membership function for the cell or pair (EC, DP).

The membership functions play the important role in the mapping function from $\{\mathbf{D O}\}$ to $\{\mathbf{R C}\}$. For each pair of $\mathrm{DO}_{i}$ and $\mathrm{RC}_{j}$, a set of membership functions should be established corresponding to the five grades of fuzzy measures: excellent, good, satisfactory, unsatisfactory, and poor.

There are a variety of membership functions, such as Gaussian distribution, normal distribution function, and trap- ezoidal function. Figure 2 shows one example. The limitation to the minimum ideal value can be described as the upper step function or S-membership function. The limitation to an ideal range can be described as trapezoidal function, П-membership function, or normal distribution function; and the limitation to the maximum ideal value can be described as the complementation of a step function or $\mathrm{S}$-membership function.

\subsection{Step 4: Aggregate with fuzzy operators}

Individual fuzzy impacts of DPs on ECs are processed to obtained intermediate and final results in the following four ways:

1. the aggregation from individual DPs to one EC,

2. the upward aggregation along the RC hierarchy,

3. the aggregation from individual ECs to one DP, and

4. the upward aggregation along the DO hierarchy.

The first occasion is the same as the third. Because the aggregation takes place along rows or columns in a matrix, it can be called a matrix (flat) aggregation. The second occasion is the same as the fourth. Because it takes place upward on a hierarchical tree, it is therefore called a hierarchical aggregation.

Let us consider the matrix flat aggregation first. The following equation is proposed for the flat aggregation in a matrix:

$$
\text { row }_{i}=\sum_{j=1}^{n} \text { cell_weight }_{i, j} \times \text { column_weight }_{j}
$$

for aggregating along the $n$ columns in the matrix and

$$
\text { column }_{j}=\sum_{i=1}^{m} \text { cell_weight }_{i, j} \times \text { row_weight }_{i}
$$

for aggregating along the $m$ rows in the matrix.

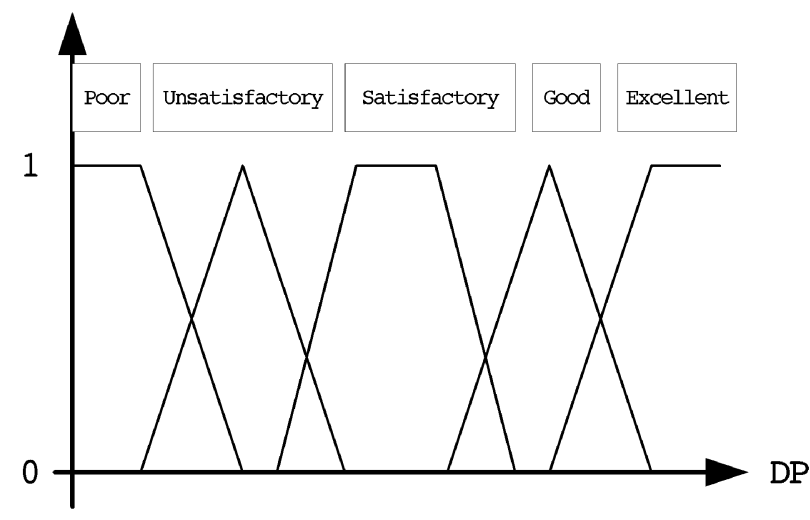

Fig. 2. Typical membership functions. 
Row $_{i}$ and Column ${ }_{j}$ are aggregated weights (results) for the $i$ th row and $j$ th column of the matrix, respectively. They

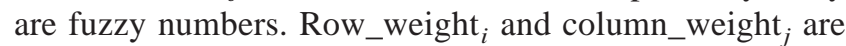
input weights assigned to the $i$ th row and $j$ th column of the matrix, respectively. These weights can be fuzzy numbers. However, they are also assumed to be crisp numbers in the above equations if normalized. If these weights are empty, they are omitted from the computation in the above equations. Cell_weight ${ }_{i, j}$ is of course the fuzzy number obtained from the membership function corresponding to the $i$ th row and $j$ th column of the matrix. Empty cell_weight ${ }_{i, j}$ is omitted from the computation using the above two equations.

Now let us move on to the issue of hierarchical aggregation. Let us assume that an element in the tree has $k$ immediate lower level elements. Let higher denote the fuzzy weight of the higher element, and $\{$ lower $\}$ and $\{$ weight $\}$ denote the set of fuzzy weights and normalized weights of the lower level elements, respectively. Thus, higher can be evaluated as follows

$$
\text { higher }=\sum_{i=1}^{k} \text { weight }_{i} \times \text { lower }_{i} .
$$

The above methods for both the matrix and hierarchical aggregations are very simple but widely used in practice. There are 15 fuzzy operators that are available for processing fuzzy numbers (Mizumoto, 1982). These operators can be used to form more sophisticated aggregation methods as has been done in the literature. For example, the new fuzzy weighted average method was recently proposed for hierarchical aggregation (Vanegas, 1999; Vanegas \& Labib, 2001a).

\subsection{Step 5: Defuzzificate the results and observe for further investigations}

The individual, intermediate, or final results from the aggregations are fuzzy numbers. They must be properly interpreted in order to draw meaningful conclusions. To defuzzify a group of fuzzy numbers, it is important to consider the method sensitivity to the variations in the fuzzy numbers for the most possible cases and its ability to discriminate among the greatest number of fuzzy numbers. Several strategies are commonly used for defuzzification (Chen \& Klein, 1997):

- the weighted center of a fuzzy number (Kim \& Park, 1990),

- the area center of fuzzy number (Chen \& Klein, 1997),

- the total integral value of a fuzzy number (Liou \& Wang, 1992),

- the left and right assigned cores of a fuzzy number (Chen et al., 1992), and

- the $\alpha$-cut and fuzzy subtraction operation (Chen \& Klein, 1997).

At present, very simple defuzzification methods are used in our case studies and the suitability of the above strategies are yet to be investigated. Generally speaking, the biggest measure in the fuzzy set is the ultimate decision. For example, if the fuzzy set is $\{0.738,0.913,0.943\}$, corresponding to \{excellent, satisfactory, poor , then the implication is that this design is poor because 0.934 is the biggest measure and corresponds to the linguistic term poor. The centroid method is also used in our case studies.

Various analyses can be carried out based on the results obtained in the preceding steps:

- The overall decision (excellent, good, satisfactory, unsatisfactory, poor) concerning DR can be obtained for the overall design.

- It is possible to find the strengths and weaknesses of the design by looking at the intermediate results in terms of the review viewpoints in the RC hierarchy.

- It is possible to ascertain the strengths and weaknesses of a design by looking at intermediate results in terms of the higher level DOs in the DO hierarchy.

- Opportunities for improvement of the redesign of the design under review can be identified with respect to overall and/or individual RCs.

The first three applications are basically the same. Individual results are obtained by flat aggregations along the rows and columns in the review matrix. Individual results are then aggregated up the DO and RC hierarchies as necessary, leading to intermediate results. The hierarchical aggregations stop when the tops of the hierarchies are reached, resulting in the overall results. In theory, the overall results obtained from the DO hierarchy and the RC hierarchy should be more or less the same, representing the overall result of the DR.

The fourth application of optimizing the DPs in the $\{\mathbf{D P}\}$ domain is possible. This is beyond the scope of this paper and will be discussed separately in the near future. Its principle of design optimization is based on the work originally proposed by Vanegas and Labib (2001b), as shown in Figure 3 . It is briefly summarized as follows:

1. The focus of optimization (i.e., the objective function) must be identified in the RC hierarchy. The choice solely depends on the objective of the analyst.

2. The DPs of particular concern $\{\mathbf{D P}\}_{k}$ are identified from the entire $\{\mathbf{D P}\}$ domain, $\{\mathbf{D P}\}_{k} \subseteq\{\mathbf{D P}\}$.

3. For each $\mathrm{DP}_{i}$ in $\{\mathbf{D P}\}_{k}, \mathrm{DP}_{i} \in \mathrm{VR}_{i}$, where $\mathrm{VR}_{i}$ is the value range corresponding to $\mathrm{DP}_{i}$.

3a. Calculate the criterion satisfaction weights $\mathrm{RW}_{i}$.

$3 \mathrm{~b}$. Plot the results in a diagram as shown in Figure 3.

3c. The peak point in the diagram is identified, and the corresponding value is the optimum choice from the chosen objective function.

4. Step 2 is repeated for all the chosen DPs.

\section{CASE STUDY: BUS INNER CONFIGURATION}

This case study is concerned with the DR of the bus inner configuration. The design was created in the CAD (Pro/ 


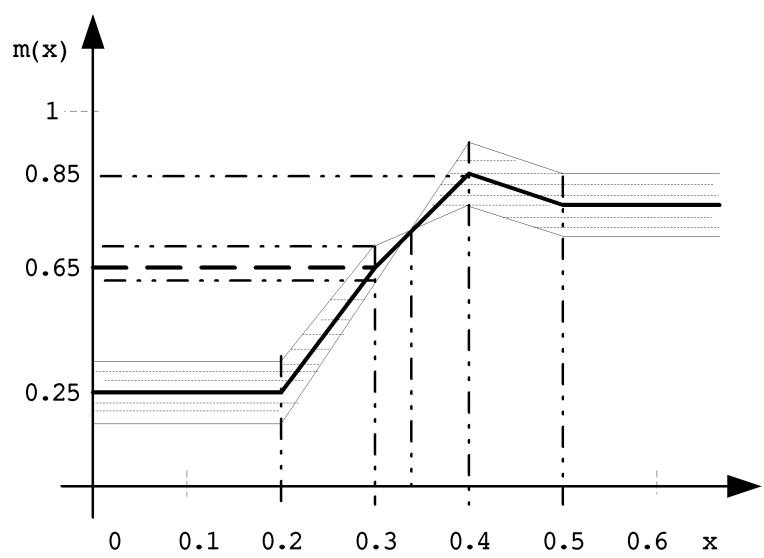

Fig. 3. Optimizing the value of a design parameter in terms of a review criterion.

Engineer) environment and then converted into the VRML format using the virtual reality environment (i.e., the ERGO module of DENEB software). The driver workspace part of the design is shown in Figure 4, while the rest is omitted for confidentiality reasons. Because the bus was designed for Chinese drivers, this VRML design model is used for simulation with the data for Chinese manikins in order to evaluate the performance of the design related to ergonomics.

The design includes several components such as driver seat, passenger seat, handrail, instrument panel, steering wheel, foot board, and operation bar. For each component, key design attributes or parameters can be identified. They are listed in Table 1 and partially displayed in Figure 4, together with their values. This is the $\{\mathbf{D P}\}$ domain in FuzzySTAR. The normalized weights of DPs are also given in the table.

One point deserves explanation. The operation bar and steering wheel are actually separate parts. However, their

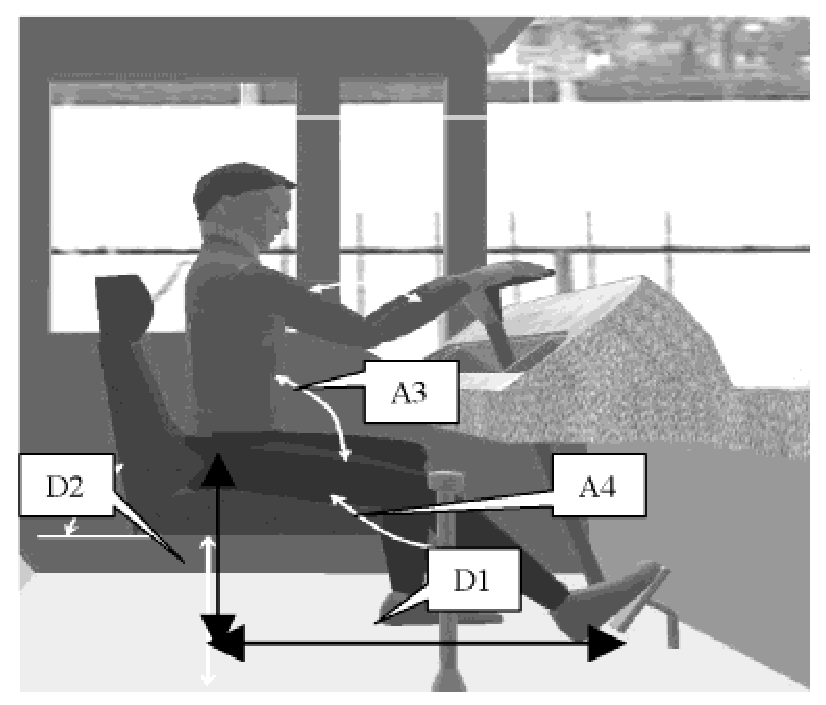

Fig. 4. Ergonomics measure in the virtual reality environment.

three parameters $\mathrm{L} 1, \mathrm{~A} 1$ and $\mathrm{A} 2$ are treated within the same group when assigning the weightings. The same point is made for parameters D1, A3, D2, and A4 of foot board and driver seat.

A series of DRs have been conducted for the bus inner configuration designs from various points of view, for example, cost, manufacturability, ergonomics, and aesthetics. This case study, however, focuses on the DR with respect to ergonomics of the bus inner configuration. Specific criteria established for the DR in this case study include driver accessibility, driver seating convenience, passenger seating convenience, and handrail location rationality. They are listed in Table 2. Their normalized weights are also given, based on consultation with experts in the field. These weights will be used later for hierarchical aggregation in this $\{\mathbf{R C}\}$ domain of FuzzySTAR.

Table 1. $\{$ DO $\}$ domain: Design parameters, weights, and values

\begin{tabular}{lclcc}
\hline \hline Part Name & DP Symbol & \multicolumn{1}{c}{ Description } & Weights & Value \\
\hline Operation bar & L1 & Length of operation bar $(\mathrm{cm})$ & 0.25 & 55 \\
& A1 & Angle of operation bar $\left(^{\circ}\right)$ & 0.3 & 87 \\
Steering wheel & A2 & Normal direction of steering wheel $\left(^{\circ}\right)$ & 0.45 & 72 \\
Passenger seat & A5 & Angle of leg and upper part of body $\left(^{\circ}\right)$ & 0.2 & 113 \\
& D3 & Distance from knee to forward row $(\mathrm{cm})$ & 0.3 & 5.5 \\
& A6 & Angle of leg and shank $\left(^{\circ}\right)$ & 0.2 & 114 \\
Foot board & D4 & Distance from point H to ground board in bus $(\mathrm{cm})$ & 0.3 & 39 \\
Driver seat & D1 & Horizontal distance from foot board to H point in body $(\mathrm{cm})$ & 0.2 & 58 \\
& A3 & Angle of leg and upper part of body $\left(^{\circ}\right)$ & 0.3 & 108 \\
& D2 & Distance from point H to ground board in bus $(\mathrm{cm})$ & 0.2 & 39.5 \\
Handrails & A4 & Angle of leg and shank $\left(^{\circ}\right)$ & 0.3 & 130 \\
& H1 & Height of handrail $(\mathrm{cm})$ & 0.667 & 180 \\
& D5 & Offset distance from side of bus $(\mathrm{cm})$ & 0.333 \\
\hline \hline
\end{tabular}


Table 2. $\{\mathbf{R C}\}$ domain review criteria and weights

\begin{tabular}{lllc}
\hline \hline Viewpoint & RC Symbol & \multicolumn{1}{c}{ Description } & Weight \\
\hline Ergonomics & D-A & Driver accessibility & 0.20 \\
& P-S-C & Passenger seating convenience & 0.25 \\
& D-S-C & Driver seating convenience & 0.25 \\
& H & Handrail location rationality & 0.30
\end{tabular}

Having defined the two $\{\mathbf{D P}\}$ and $\{\mathbf{R C}\}$ domains, it is necessary now to establish fuzzy membership functions between the elements in $\{\mathbf{D P}\}$ and elements in $\{\mathbf{R C}\}$. In this case study, there are potentially $13 \times 4=52$ membership functions that have to be established. In order to minimize the efforts, an initial task has been carried out to determine if a particular DP affects a given RC. If not, there is no need to establish the membership function between them. This DP therefore belongs to the uncaring-type DP with respect to this RC. If a certain relationship is identified, further work is done to establish the membership function between them.

After the initial screening, the following DP-RC relationships were observed:

- For driver accessibility, we consider the length and angle of the operation bar and the normal direction of the steering wheel.

- For driver seating convenience, we consider the angle of the leg and upper part of body, the angle of the leg and shank, the horizontal distance from foot board to point $\mathrm{H}$ in the body, and the distance from point $\mathrm{H}$ to the ground board in the bus.

- For passenger seating convenience, we consider the angle of the leg and upper part of body, the angle of the leg and shank, the distance from the knee to the row in front, and the distance from point $\mathrm{H}$ to the ground board in the bus.

- For the handrail location rationality, we consider the height of the handrail and the offset distance from the side of the bus.

Tables 3-7 show some of the membership functions established for $\{\mathbf{D P}\}$ and $\{\mathbf{R C}\}$ used in this case study. The membership functions to the ranks unsatisfactory and poor are the complements of good and excellent, respectively. The values of $\alpha, \beta, \gamma$, and $\lambda$ are derived from the Chinese national regulations and by consulting engineers of the case company. Because of the lack of experience, the trapezoidal and step membership function are used. Most RCs are limited to an ideal range or minimum.

Figure 5 shows the FuzzySTAR worksheet completed for the case study. As can be seen, this worksheet clearly reflects the logic of the FuzzySTAR methodology outlined in Section 2. Therefore, it is straightforward to interpret the results in the worksheet.

For example, when L1 is set as $55(\mathrm{~cm})$, we have its mapping result to driver accessibility (D-A) is $\{0,0,0.167\}$ after referring the trapezoidal membership function of L1 to the criteria D-A. They correspond to the ranks excellent, good, and satisfactory, respectively. Likewise, we have the mapping set $\{0,0,0.167,1,1\}$ from L1 to D-A. Similarly, we have the mapping set $\{1,0.75,0.667,0.25,0\}$ from A1 to D-A and $\{1,1,0.8,0,0\}$ from $\mathrm{A} 2$ to $\mathrm{D}-\mathrm{A}$. The process of such fuzzy mapping is conducted until all the cells in the review space have been considered.

The next step is to aggregate the individual mapping results along each row (i.e., $\mathrm{RC}$ ). For the $\mathrm{RC}=\mathrm{D}-\mathrm{A}$, three fuzzy numbers have been obtained. They are $\{0,0,0.167,1$, $1\},\{1,0.75,0.667,0.25,0\}$, and $\{1,1,0.8,0,0\}$. They are synthesized to obtain the overall weight for the corresponding row in the worksheet as follows:

$$
\begin{gathered}
0.3 \quad 0.45) \circ\left[\begin{array}{ccccc}
0 & 0 & 0.167 & 1 & 1 \\
1 & 0.75 & 0.667 & 0.25 & 0 \\
1 & 1 & 0.8 & 0 & 0
\end{array}\right] \\
\quad=\left(\begin{array}{lllll}
0.75 & 0.675 & 0.602 & 0.325 & 0.25
\end{array}\right) .
\end{gathered}
$$

After normalization, the review result fuzzy number becomes

$$
\{0.288,0.259,0.231,0.125,0.096\}
$$

Similarly, the overall weights for other RCs can be obtained. All the results are presented in the corresponding column of the worksheet.

Next, the hierarchical aggregation is conducted. In order to do this, the importance ratings must be assigned to the

Table 3. Trapezoidal membership function coefficients between $D-A$ and $\{L 1, A 1, A 2\}$

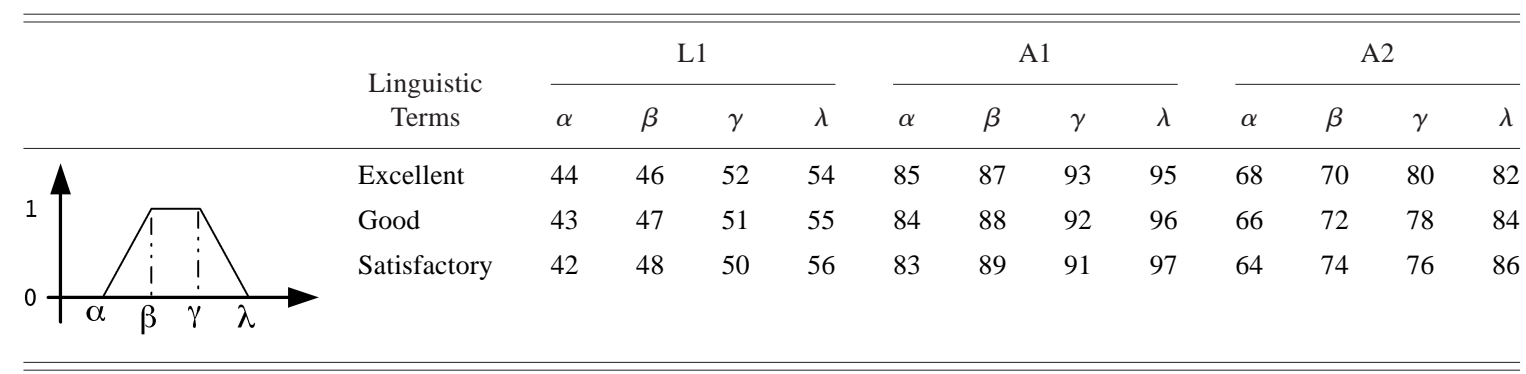


Table 4. Upper step membership function coefficients between $P-S-C$ and $\{A 5, D 3, A 6\}$

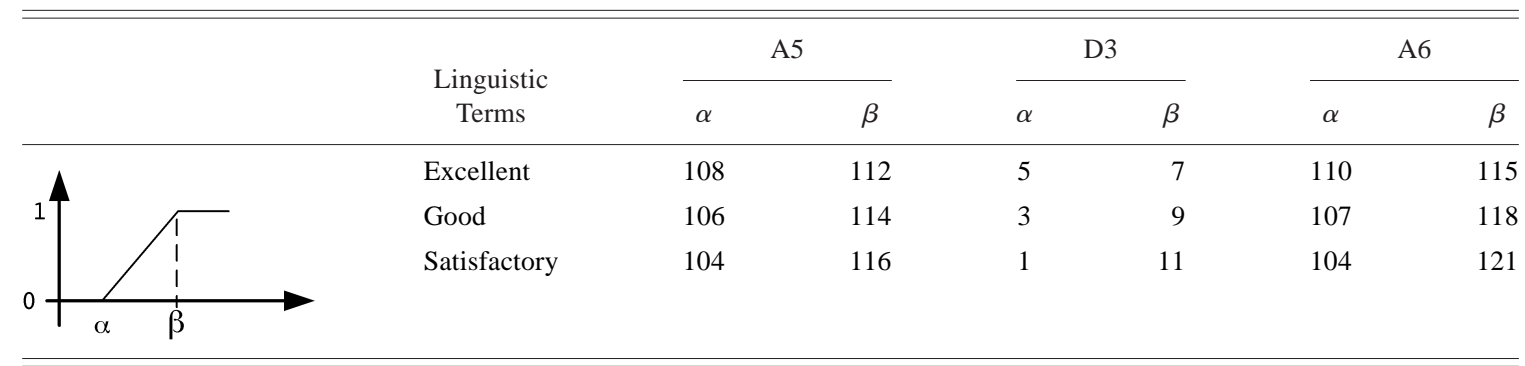

individual RCs. The hierarchical aggregation process is conducted as follows:

$$
\begin{gathered}
\left(\begin{array}{llllll}
0.2 & 0.25 & 0.25 & 0.3
\end{array}\right) \\
\circ\left[\begin{array}{cccccc}
0.288 & 0.259 & 0.231 & 0.125 & 0.096 \\
0.23 & 0.227 & 0.217 & 0.167 & 0.159 \\
0.346 & 0.292 & 0.274 & 0.072 & 0.016 \\
0.215 & 0.206 & 0.206 & 0.188 & 0.185
\end{array}\right] \\
\\
\quad=\left(\begin{array}{llllll}
0.2661 & 0.2433 & 0.2307 & 0.1411 & 0.1184
\end{array}\right) .
\end{gathered}
$$

After normalization, the overall weight for the viewpoint ergonomics is $(0.281,0.254,0.238,0.127,0.1)$.

Finally, we have reached a point to address the question of whether the design is good or bad. Let us observe the largest numbers in the fuzzy sets of the overall weights for the design. Apparently, the design is excellent because this grade has the largest number in the fuzzy set. The difference between the excellent and good numbers is $\Delta=0.281-$ $0.254=0.027$, that is, $0.027 / 0.254 \times 100 \% \approx 10 \%$. Therefore, this level of confidence of deriving the decision that the design is excellent is reasonably acceptable.

When individual RCs are examined in a similar way, it can be seen that all of them are excellent. After defuzzification using the centroid method, their crisp numbers are $\{0.704,0.638,0.777,0.613)$ in ranked order corresponding to $\{\mathrm{D}-\mathrm{S}-\mathrm{C}, \mathrm{D}-\mathrm{A}, \mathrm{P}-\mathrm{S}-\mathrm{C}, \mathrm{H})$ respectively. Because all of them are excellent and the differences between them are not sig-

Table 5. Trapezoidal membership function coefficients between $P-S-C$ and $D 4$

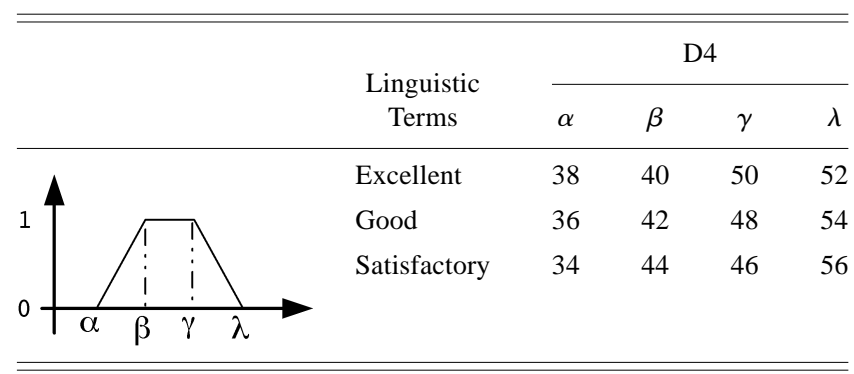

nificant, no redesign actions are recommended to improve the ergonomics.

\section{CONCLUDING DISCUSSION}

This paper has presented a novel approach to design review. This approach integrates fuzzy set theory with STAR, which we proposed in our previous research (Huang, 2002a, $2002 b$ ). The resulting FuzzySTAR provides not only a powerful methodology but also a practical tool for both the product design team and the DR team to use to institutionalize a formal design mechanism in the product development process.

In FuzzySTAR, DR is defined as the reverse mapping between the DP domain and design requirement $(\mathrm{RC})$ domain, as compared with Suh's TAD. This definition implies that both the design team and the review team share the same workspace, which is characterized by the DP and the design requirement ( $\mathrm{RC}$ ) domains. This minimizes the amount of extra preparatory work as required in other DR approaches and methods.

Fuzzy sets are extensively introduced in the definitions of the domains and the mapping process to deal with imprecision, uncertainty, and incompleteness in product definition data and expert judgments. Both the DP and RC domains are represented hierarchically. Fuzzy weights are assigned to each level using the fuzzy AHP. These weights are used for aggregating the fuzzy estimates from the lower to upper levels of the hierarchies until the top level is reached.

The impacts of DPs on RCs are also described in fuzzy numbers through membership functions based on previous experience and technical judgments. Individual impacts are then aggregated along the rows and/or columns for the individual RCs and/or DPs, respectively. These individual intermediate fuzzy measures are themselves used for identifying areas where the design is good or requires improvement, as well as used for aggregating to obtain overall measures for overall decisions.

The simple case study has been extracted from a comprehensive industrial problem to demonstrate how FuzzySTAR can be applied in real-life industrial design projects. In the meanwhile, this case study also highlights a few lim- 
Table 6. Trapezoidal membership function coefficients between $D-S-C$ and $\{A 3, D 1, D 2, A 4\}$

\begin{tabular}{|c|c|c|c|c|c|c|c|c|c|c|c|c|c|c|c|c|c|c|}
\hline & & \multirow{2}{*}{$\begin{array}{l}\text { Linguistic } \\
\text { Terms }\end{array}$} & \multicolumn{4}{|c|}{$\mathrm{A} 3$} & \multicolumn{4}{|c|}{ D1 } & \multicolumn{4}{|c|}{ D2 } & \multicolumn{4}{|c|}{ A4 } \\
\hline & & & $\alpha$ & $\beta$ & $\gamma$ & $\lambda$ & $\alpha$ & $\beta$ & $\gamma$ & $\lambda$ & $\alpha$ & $\beta$ & $\gamma$ & $\lambda$ & $\alpha$ & $\beta$ & $\gamma$ & $\lambda$ \\
\hline . & & Excellent & 97 & 99 & 109 & 111 & 50 & 52 & 58 & 60 & 38 & 40 & 50 & 52 & 126 & 128 & 134 & 136 \\
\hline 1 & & Good & 95 & 101 & 107 & 113 & 49 & 53 & 57 & 61 & 36 & 42 & 48 & 54 & 125 & 129 & 133 & 137 \\
\hline & & Satisfactory & 93 & 103 & 105 & 115 & 48 & 54 & 56 & 62 & 34 & 44 & 46 & 56 & 124 & 130 & 132 & 138 \\
\hline
\end{tabular}

itations that further research must overcome. First, the values of DPs are at present crisp. That is, design decisions are certain and accurate. This is unlikely to be the case if the design is at the early stages such as concept design. FuzzySTAR should be made adequately flexible to deal with both the crisp and fuzzy DPs.

Second, it is assumed at present that the impact of one DP on one RC is independent of other DPs and RCs. This assumption, although a reasonable and acceptable simplification, is not consistent with the principle of modular design, whereby a module is a well-established design including a set of DPs for achieving a set of multiple design requirements. If such modular combinations are identified in a design, extra merit should be assigned during the DR process.

Third, the absolute impacts of DPs on RCs are not estimated or measured during the fuzzy DR process. For example, the cost of design is evaluated as expensive, affordable, or cheap without specifying the actual amount in dollar terms. In some real-life industrial design projects, absolute measurements are necessary, as are the fuzzy estimates.

Fourth, the simple case study illustrated that the final outcomes are very sensitive to the constructs inherent in fuzzy set theory. The definition of meaningful membership functions is essential. The choice of appropriate fuzzy operators in various aggregations affects the final results significantly. Fuzzy weights assigned to DP and RC hierarchies are extremely sensitive to the final results. Great care should be taken when determining these weights, and rigorous methods should be followed to capture experts' judgments. The defuzzification strategies also lead to variations of final re- sults. All these aspects must be carefully verified and refined before a fuzzy DR system can be truly applied to solve design problems in an industrial environment.

Fifth, STAR and FuzzySTAR do share some degree of similarity with quality function deployment (QFD; Hauser \& Clausing, 1988) and fuzzy QFD (Khoo \& Ho, 1996; Vanegas \& Labib, 2001a), respectively. Such similarity exists in terms of not only formality but also the contents to some extent. The horizontal dimension in STAR and FuzzySTAR is the same as that of QFD and FuzzyQFD, representing the design features or characteristics. If the design is reviewed from the point of view of the customers, then their requirements can be directly used as $\{\mathbf{R C}\}$ in STAR and FuzzySTAR. However, STAR and FuzzySTAR accommodate other types of algorithms for evaluating the impacts of $\{\mathbf{D P}\}$ on $\{\mathbf{R C}\}$ especially in the tandem approach (Huang \& Jiang, 2002b), in addition to the methods used in QFD and FuzzyQFD. Another distinction is that design capabilities $\{$ DC $\}$ are usually quantitatively evaluated from STAR and FuzzySTAR.

Sixth, in the context of a DR portal, as proposed previously (Huang, 2002b), FuzzySTAR is only one method commonly shared by all the members in the DR team, that is, only one of the so-called common review forms. It is not intended to replace, but rather to complement, other disciplinespecific DR methods.

Seventh, the design in the case study happens to be a good design. If the design turns out to be unsatisfactory, efforts should be made to identify what design features cause the problem in terms of which RCs and how the problem

Table 7. Trapezoidal membership function coefficients between $H$ and $\{H 1, D 5\}$

\begin{tabular}{|c|c|c|c|c|c|c|c|c|c|}
\hline & \multirow{2}{*}{$\begin{array}{c}\text { Linguistic } \\
\text { Terms }\end{array}$} & \multicolumn{4}{|c|}{$\mathrm{H} 1$} & \multicolumn{4}{|c|}{ D5 } \\
\hline & & $\alpha$ & $\beta$ & $\gamma$ & $\lambda$ & $\alpha$ & $\beta$ & $\gamma$ & $\lambda$ \\
\hline \multirow{3}{*}{14} & Excellent & 172 & 174 & 179 & 181 & 8 & 9 & 14 & 15 \\
\hline & Good & 171 & 175 & 178 & 182 & 7 & 10 & 13 & 16 \\
\hline & Satisfactory & 170 & 176 & 177 & 183 & 6 & 11 & 12 & 17 \\
\hline
\end{tabular}




\begin{tabular}{|c|c|c|c|c|c|c|c|c|}
\hline \multicolumn{2}{|c|}{ Cost } & \multicolumn{4}{|c|}{ Ergonomics } & & & \\
\hline & & \multicolumn{4}{|c|}{$(0.281,0.254,0.238,0.127,0.1)$} & & & \\
\hline & & (H) & (D-S-C) & $(\mathrm{P}-\mathrm{S}-\mathrm{C})$ & (D-A) & & & \\
\hline & & 0.3 & 0.25 & 0.25 & 0.2 & & & \\
\hline & & $(0.215,0.206,0.206,0.188,0.185)$ & $(0.346,0.292,0.274,0.072,0.016)$ & $(0.23,0.227,0.217,0.167,0.159)$ & $0.288,0.259,0.231,0.125,0.096)$ & & & \\
\hline & 8 & & & & $(0,0,0.167,1,1)$ & 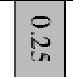 & $=$ & Operation \\
\hline & * & & & & $(1,0.75,0.667,0.25,0)$ & 8 & $\geq$ & bar \\
\hline 8 & $*$ & & & & $(1,1,0.8,0,0)$ & $D_{1}^{\infty}$ & 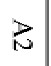 & Steer wheel \\
\hline$*$ & & & & $(1,0.875,0.75,0.125,0)$ & & in & 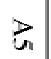 & \\
\hline$*$ & & & & $(0.25,0.416,0.45,0.584,0.75)$ & & 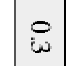 & $\Xi$ & Passenoer \\
\hline+ & + & & & $(0.8,0.636,0.588,0.364,0.2)$ & & i & a & seat \\
\hline & $\$$ & & & $(0.5,0.5,0.5,0.5,0.5)$ & & $\stackrel{\omega}{\omega}$ & 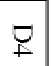 & \\
\hline & & & $(1,0.75,0.667,0.25,0)$ & & & i & $\underline{\Xi}$ & $\begin{array}{l}\text { Foot- } \\
\text { board }\end{array}$ \\
\hline & & & $(1,0.833,0.7,0.167,0)$ & & & $\stackrel{\omega}{\omega}$ & 己 & \\
\hline$*$ & & & $(0.75,0.583,0.55,0.417,0.25)$ & & & is & 怘 & $\begin{array}{c}\text { Driver } \\
\text { seat }\end{array}$ \\
\hline 8 & & & $(1,1,1,0,0)$ & & & $\ddot{\omega}$ & 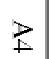 & \\
\hline 8 & & $(0.5,0.5,0.5,0.5,0.5)$ & & & & $\bar{\alpha}$ & $\Xi$ & \\
\hline & & $(0.6,0.53,0.52,0.467,0.4)$ & & & & $\bar{\omega}$ & 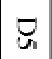 & 14. \\
\hline
\end{tabular}

Fig. 5. The worksheet of FuzzySTAR for the case study on a bus inner configuration design. 
can be rectified. Based on the FuzzySTAR final and intermediate results, potential areas for improvement are usually identified and redesign advice is indicated as well. If the fuzzy evaluations are conducted over the whole value ranges of DPs, trends of the impact on the chosen RCs can be observed. Such trends indicate the directions of adjusting the values of particular DPs in order to optimize the RCs. This motivates us to research into a fuzzy design revision methodology and system. In fact, the DR with STAR and FuzzySTAR is considered only as a single step for calculating the fitness in an evolutionary design process (Huang \& Jiang, 2002b). In this sense, the evolutionary design refinement is outside the scope of the FuzzySTAR framework.

Eighth and finally, the word axioms has not been explicitly dealt with in this paper. Initial efforts have been made to examine what roles the axioms and theorems proposed in Suh's TAD can play in DR. A cautious and preliminary finding is that they are generally applicable and require little extension. It remains a great challenge to determine whether fuzzy axioms and theorems can be identified and established.

The uses of the two design axioms (independence and information) in DR are not examined in this work. One reason is that the independence axiom is assumed to be tested and followed during the process of generating the good design solution submitted for review. The information axiom is introduced in our nonfuzzy version of our work on DR. The information is defined as the difference between the design requirements and DCs sets. The smaller this difference, the fitter the design is. This is used as a method for evaluating the fitness function of our evolutionary design (Huang \& Jiang, 2002a) framework. In-depth discussions on the roles of axioms in DR are outside the scope of this paper. In fact, a separate paper (Huang, 2002a) attempts to examine the roles and relevance of the two well-known design axioms in the context of DR. There is no doubt that this is an area that deserves further investigation.

\section{ACKNOWLEDGMENTS}

The authors are most grateful to the Hong Kong Research Grant Council, Hong Kong University Committee on Research and Conference Grants, and Hong Kong University William Mong Engineering Foundation for financial support that made this research possible. We would also like to thank the two referees for their critical and constructive comments.

\section{REFERENCES}

Carnahan, J.V., Thurston, D.L., \& Liu, T. (1994). Fuzzing ratings for multiattribute design decision-making. Journal of Mechanical Design, Transactions of the ASME 116, 511-521.

Cha, S.W., \& Cho, K.K. (1999). Development of DVD for the next generation by axiomatic approach. CIRP Annals 48, 85 .

Chen, S.J., Hwang, C.L., \& Hwang, F.P. (1992). Fuzzy Multiple Attributes Decision Making Methods and Applications. New York: Springer.
Chen, C.B., \& Klein, C.M. (1997). An efficient approach to solving fuzzy MADM problems. Fuzzy Sets and Systems 88, 51-67.

Chen, K.Z (1998). Integration of design method software for concurrent engineering using axiomatic design. International Journal of Manufacturing Technology Management 9, 242-252.

Chen, K.Z (2001). Development of integrated design for disassembly and recycling in concurrent engineering. International Journal of Manufacturing Technology Management 12, 67-79.

Cheng, C.H. (1999) Evaluating weapon systems using ranking fuzzy numbers. Fuzzy Sets and Systems 107, 25-35.

Harutunian, V., Nordlund, M., Tate, D., \& Suh, N.P. (1996). Decision making and software tools for product development based on axiomatic design theory. CIRP Annals 45(1), 135.

Hauser, J.R., \& D. Clausing (1988). The house of quality. Harvard Business Review May-June, 63-73.

Helander, M.G., \& Lin, L. (2000). Anthropometric design of workstations. Proc. First Int. Conf. Axiomatic Design, Cambridge, MA.

Hintersteiner, J.D., \& Friedman, G. (1999). System architecture template. White paper.

Huang, G.Q. (2002a). STAR: Systematic theory of axiomatic design review and web-based design review portal. Manuscript submitted for publication.

Huang, G.Q. (2002b). Web-based support for collaborative product design review. Computers in Industry $48(1)$.

Huang, G.Q., \& Jiang, Z.H. (2002a) Evolutionary product customization using genetic operators. Annals of 2002 International CIRP Design Seminar, May 16-18, 2002, Hong Kong.

Huang, G.Q., \& Jiang, Z.H. (2002b). Web-based design review of fuel pumps using fuzzy set theory. Manuscript submitted for publication.

Ichida, T. (1989). Deizain Rebyu Jireishu. Portland, OR: Productivity Press.

Khoo, L.P., \& Ho, N.C. (1996). Framework of a fuzzy quality function deployment system. International Journal of Production Research 34, 299-311.

Kim, K., \& Park, K.G. (1990). Ranking fuzzy numbers with index of optimism. Fuzzy Sets and Systems 35, 143-150.

Kim, S.-J., Suh, N.P., \& Kim, S.-G. (1991). Design of software systems based on axiomatic design. Robotics and Computer-Integrated Manufacturing 8(4), 243-255.

Li, H., \& Azarm, S. (2000). Product design selection under uncertainty and with competitive advantage. Journal of Mechanical Design, Transactions of the ASME 122, 411-418.

Liou, T.S., \& Wang, M.J. (1992). Fuzzy weighted average: An improved algorithm. Fuzzy Sets and Systems 50, 247-255.

Masud, A.S.M., \& Dean, E.B. (1993). Using fuzzy sets in quality function deployment. Proc. 2nd Industrial Engineering Research Conf., pp. 270-274.

Mizumoto, M. (1982). Comparison of fuzzy reasoning methods. Fuzzy Sets and Systems 8, 253-283.

Moskowitz, H., \& Kim, K.J. (1997). QFD optimiser: A novice friendly quality function deployment decision support system for optimising product designs. Computers and Industrial Engineering 32, 641-655.

Park, C.B., Baldwin, D.F., \& Suh, N.P. (1996). Axiomatic design of a microcellular filament extrusion system. Research in Engineering Design 8, 166-177.

Park, T., \& Kim, K.J. (1998). Determination of an optimal set of design requirements using house of quality. Journal of Operations Management 16, 569-581.

Schoonmaker, S.J. (1996). ISO 9001 for Engineering and Designers. New York: McGraw-Hill.

Suh, N.P. (1990). The Principles of Design. New York: Oxford University Press.

Suh, N.P. (1999). Axiomatic Design: Advances and Applications. New York: Oxford University Press.

Suh, N.P., Bell, A.C., \& Gossard, D.C. (1978). On an axiomatic approach to manufacturing and manufacturing systems. Journal of Engineering for Industry, Transactions of the ASME 100, 127-130.

Tate, D. (1999). "A roadmap for decomposition: Activities, theories, and tools for system design." PhD Thesis. Cambridge, MA: Massachusetts Institute of Technology, Department of Mechanical Engineering.

Vanegas, L.V. (1999). Application of fuzzy set theory in engineering design. MS dissertation. Manchester, UK: University of Manchester Institute of Science and Technology.

Vanegas, L.V., \& Labib, A.W. (2001a) Application of new fuzzy-weighted 
average (NFWA) method to engineering design evaluation. International Journal of Production Research 39, 1147-1162

Vanegas, L.V., \& Labib, A.W. (2001b). A fuzzy quality function deployment (FQFD) model for deriving optimum targets. International Journal of Production Research 39, 99-120.

Wallace, D.R., \& Suh, N.P. (1993). Information-based design for environmental problem solving. Annals of the CIRP 42, 175-179.

Wang, J. (1999). Fuzzy outranking approach to prioritise design requirements in quality function deployment. International Journal of Production Research 37, 899-916.

Wang, J., (2001). Ranking engineering design concepts using a fuzzy outranking preference model. Fuzzy Sets and Systems 119, 161-170.

George Huang is Associate Professor in the Department of Industrial and Manufacturing Systems Engineering, the University of Hong Kong. He obtained his BEng degree in Mechanical Engineering from Southeast University (China) and PhD degree in Mechanical Engineering from the University of Wales, Cardiff, UK. His main research areas include collaborative product commerce and digital manufacturing. He has published extensively on these topics, including around 50 refereed journal papers and book chapters, two monographs, and an edited reference book. Dr. Huang is on the editorial boards of several international journals and is a Chartered Engineer and a member of IEE, ASME, IIE, and HKIE.

Zuhua Jiang is Associate Professor and Associate Head of the Department of Industrial Engineering, Shanghai Jiao Tong University, where he obtained his MEng and $\mathrm{PhD}$ degrees. Dr. Jiang has been involved in a number of major research projects at the Computer Integrated Manufacturing Systems (CIMS) Institute of Shanghai Jiao Tong University in the areas of knowledge-based product design and manufacturing. He is also a visiting Research Fellow in the Department of Industrial and Manufacturing Systems Engineering of the University of Hong Kong. 